\title{
Modulation of Peripheral Chemoreflex by Neurohumoral AdAPTATIONS AFTER Kidney TRANSPlantation
}

\author{
T. Rassaf, R. Westenfeld, J. Balzer, T. Lauer, M. Merx, J. Floege, S. Steiner, C. Heiss, M. Kelm, C. Meyer \\ Division of Cardiology, Pulmonology and Angiology, University of Duesseldorf, Germany
}

\begin{abstract}
Background: Peripheral chemoreceptors residing predominantly in the carotid body monitor changes in arterial blood oxygen and are mechanistically linked to the cardiorespiratory control by the autonomic nervous system. Enhanced sympathetic activation is common in end-stage renal disease and kidney transplantation has been shown to improve cardiorespiratory reflex measures of autonomic function.

Objective: The aim of the present study was to test whether improvement in renal function following kidney transplantation is related to an improvement in chemosensory function.

Methods and Results: We compared hyperoxic chemoreflex sensitivity (CHRS) in patients after renal transplantation (RTX) to that in patients on maintenance hemodialysis (HD), and that of age- and gendermatched healthy controls. In addition, we investigated the impact of common confounding factors including pharmacological neurohumoral modulation and diabetes mellitus. The difference in the R-R intervals divided by the difference in the oxygen pressures before and after deactivation of the chemoreceptors by 5 -min inhalation of $7 \mathrm{~L}$ oxygen was calculated as the hyperoxic CHRS. Autonomic activity was characterized by 24-h time-domain heart rate variability (HRV) parameters. CHRS was improved in RTX patients as compared to HD patients being related to HRV. CHRS was related to the concomitant presence of diabetes and medication with cyclosporine.

Conclusion: Our findings indicate that chemosensory activity following kidney transplantation is related to cardiac autonomic control, but functional testing might only be useful to characterize the time course and extent of sympathetic activation in selected patients due to existing co-morbidities and immunosuppressive medication in this population.
\end{abstract}

Key words: chemoreflexes, end-stage renal disease, nervous system, autonomic

\section{INTRODUCTION}

Enhanced sympathetic activation is common in patients with end-stage renal disease (ESRD) [1-4]. Kidney transplantation has been shown to improve car- diorespiratory reflex measures of autonomic function, including heart rate variability (HRV) $[5,6]$. Peripheral chemoreceptors residing predominantly in the carotid body monitor changes in arterial blood oxygen and are also mechanistically linked to the cardiorespiratory control by the autonomic nervous system [7]. Several approaches have been proposed in order to characterize chemosenory function in both health and disease. Up to now, the desensitization characteristics of peripheral chemosensors in patients with previous kidney transplantation (RTX) has not been addressed. Therefore, the aim of the present study was to assess whether the hyperoxic chemoreflex sensitivity (CHRS), which quantifies the heart rate decrease following deactivation of chemosensors by inhalation of pure oxygen, in RTX patients differs from that in patients on maintenance hemodialysis (HD) and healthy controls.

\section{Material AND Methods \\ Study Design Patients}

The study was performed in accord with the Declaration of Helsinki for Human Research and the study protocol was approved by an institutional Ethics Committee. We studied the impact of chemosensors' deactivation on heart rate by 5 -min inhalation of $100 \%$ oxygen in RTX patients, patients on maintenance HD, and age and gender matched healthy controls. In addition, we assessed whether autonomic function, as measured by time-domain HRV parameters, is related to chemosensors' desensitization characteristics following kidney transplantation.

Chemosensory function in 19 RTX patients, $10 \mathrm{pa}-$ tients who had been on chronic HD for at least 6 months, and 10 age and gender matched healthy controls (age: $51 \pm 7$ years, 7 male, 4 smokers) were investigated. The RTX patients had been on HD previously (1-4 years). Other cardiovascular risk factors and preexisting cardiovascular disease did not preclude from participation in the study. Exclusion criteria were coexisting neurological deficit that alters autonomic function, congestive heart failure with a cardiac ejection fraction of $<35 \%$, chronic pulmonary diseases, neurocardiogenic syncope, sleep apnoea syndrome, hyperthyroidism, and heart rhythm other than sinus. 
Measurements of Heart Rate, Heart Rate

VAriability, ANd Clinical BloOd Parameters

Heart rate was measured continuously by a 12 -channel electrocardiogram (ECG). Additionally, the mean R-R interval was calculated from 10 consecutive R-R intervals. HRV time-domain measures from an ambulatory 24-h ECG were determined in $10 \mathrm{HD}$ patients performed in the interdialytic days and in 10 RTX patients. The following HRV parameters, which are considered to be standardized in the general population, were calculated for each patient to characterize cardiac sympathetic-parasympathetic control: (1) the 24-h SD of normal R-R intervals (SDNN, ms), as a global measure of HRV; (2) the SD of the average normal R-R interval for all 5-min segments of a $24-\mathrm{h}$ ECG recording (SDANN, ms); (3) the square root of the mean of the squared differences between adjacent normal R-R intervals (RMSSD, ms); and (4) the Triangular index, a time-domain geometric measure. Blood was drawn after a $30 \mathrm{~min}$ resting period through large-bore angiocatheters (18 gauge) to prevent iatrogenic hemolysis. The first $3 \mathrm{ml}$ of blood was discarded, and the blood then slowly drawn into prechilled tubes. Standard clinical blood parameters including renal function were immediately analyzed in a central laboratory using standard techniques $[8,9]$.

\section{Chemosensory Deactivation}

To characterize the effect of chemosensors' deactivation on heart rate and oxygen saturation, we determined the venous partial oxygen pressure $\left(\mathrm{PO}_{2}\right)$, before and after breathing oxygen via a nose mask. Baseline measurements were taken after patients were re- cumbent for a period of $10 \mathrm{~min}$. Pure oxygen was then administered via a nose mask for 5 min. Oxygen saturation was monitored with a pulse oximeter. Partial oxygen pressure was determined using a blood gas analyser (Radiometer Copenhagen).

The difference in the R-R intervals in the ECG before and after inhalation of pure oxygen divided by the difference in the oxygen pressures were calculated as the CHRS $(\mathrm{ms} / \mathrm{mmHg})$. A CHRS below 3.0 $\mathrm{ms} / \mathrm{mmHg}$ was defined as pathological as reported earlier [7, 10,11]. Measurements were performed after an overnight fasting period in a noise protected room of constant temperature.

\section{STATISTICAL ANALYSIS}

Data are presented as means $\pm \mathrm{SE}$ for continuous variables or as a percentage of patients for categorical variables. After testing for normal distribution, group comparisons were calculated using Student $t$-tests or Mann-Whitney U-tests for continuous variables as appropriate. Categorical data were analyzed by $\mathrm{Chi}^{2}$ with Fisher's exact test. Correlations were determined with regression (r) analysis coefficient. Multivariate analysis for CHRS prediction was assessed using the multiple regression models. A two-sided P-value $<0.05$ was considered significant. Statistical analysis was done with SPSS software for Windows version 14.0 (SPSS Inc., Chicago, IL). This trial is registered with ClinicalTrials.gov, number NCT00794872.

\section{RESULTS}

Patient characteristics are presented in Table 1. Age, gender, and cardiovascular risk factors including

Table 1. Demographic and clinical data.

\begin{tabular}{lcc}
\hline Baseline characteristics & $\begin{array}{c}\text { HD Patients } \\
(\mathrm{n}=10)\end{array}$ & $\begin{array}{c}\text { RTX Patients } \\
(\mathrm{n}=19)\end{array}$ \\
\hline Age, yr & $57 \pm 4$ & $58 \pm 3$ \\
Male sex, $\mathrm{n}$ & 7 & 12 \\
Hx hypertension, $\mathrm{n}$ & 7 & 11 \\
Hx dyslipidemia, $\mathrm{n}$ & 6 & 10 \\
Hx smoking, $\mathrm{n}$ & 6 & 5 \\
Hx CVD, $\mathrm{n}$ & 5 & 10 \\
Hx Diabetes, $\mathrm{n}$ & 2 & 2 \\
Serum creatinine, mg/dl & $7.6 \pm 0.7$ & $1.8 \pm 0.2$ \\
Blood urea nitrogen, mg/dl & $112 \pm 5$ & $72 \pm 10$ \\
\hline Medication, $\mathrm{n}$ (\%) & & 1 \\
\hline Alpha-blockers & 3 & 15 \\
Beta-blockers & 6 & 11 \\
ACE-inhibitors/AT-II-antagonists & 6 & 2 \\
Calcium antagonists & 5 & 6 \\
Central sympatholytic agents & 3 & 10 \\
Diuretics & 5 & 10 \\
Cyclosporine & 0 & 9 \\
Tacrolimus & 0 & \\
\hline
\end{tabular}

RTX, patients after kidney transplantation; HD, patients on maintenance hemodialysis; $\mathrm{Hx}$, indicates history of; CAD, coronary artery disease; CVD, cardiovascular disease; Values are means $\pm \mathrm{SE}$. 
smoking, arterial hypertension and dyslipidemia did not differ between HD patients, RTX patients, and healthy controls. Subgroup analysis did not reveal significant differences with respect to concomitant medication (alpha-blockers, beta-blockers, Ca-antagonists, and statines), beside immunosuppresion between HD and RTX patients. Basic renal diseases included nephrosclerosis $(\mathrm{n}=4)$, polycystic kidney disease ( $\mathrm{n}=$ $4)$, pyelonephritis $(n=2)$, suspected glomerulonephritis $(\mathrm{n}=3)$, and hypertensive/vascular renal damage $(\mathrm{n}=15)$. The average dialysis vintage was $32 \pm 11$ months. None of the patients had received blood transfusions during the preceding 6 months.

\section{Chemosensory Deactivation}

None of the patients were hypoxemic at baseline or after oxygen inhalation, with oxygen saturation values ranging from 95 to $100 \%$. Baseline oxygen saturation and heart rate in RTX patients were not significantly different from those observed in HD patients and healthy controls. After chemoreflex deactivation by inhalation of $100 \%$ oxygen the venous $\mathrm{PO}_{2}$ of all subjects increased while the R-R interval increased.

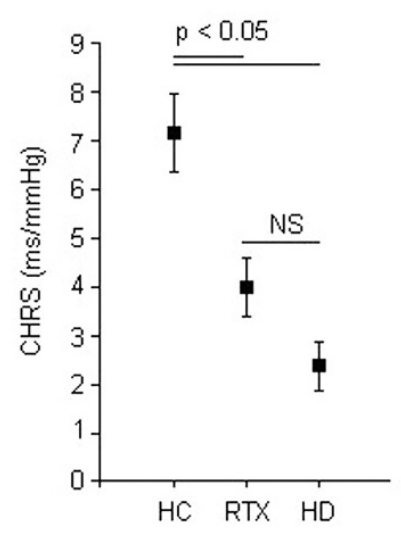

Fig. 1. Impairment of hyperoxic chemoreflex sensitivity (CHRS) in both HD and RTX patients as compared with healthy controls (HC). In addition, CHRS tended to be higher in RTX patients as compared with HD patients. Values are means \pm SE.

\section{Hyperoxic Cardiac Chemoreflex Sensitivity}

CHRS was impaired in both HD $(2.1 \pm 0.5 \mathrm{~ms} / \mathrm{mmHg})$ and RTX patients $(3.7 \pm 0.6 \mathrm{~ms} / \mathrm{mmHg})$ as compared with healthy controls $(6.9 \pm 0.8 \mathrm{~ms} / \mathrm{mm} \mathrm{Hg}$; $\mathrm{P}<0.05$, ANOVA; Fig. 1). In addition, CHRS tended to be higher in RTX patients as compared with HD patients, but this observation did not reach statistical significance $(\mathrm{P}=0.07)$.

A CHRS below $3 \mathrm{~ms} / \mathrm{mmHg}$ was found in 8 out of the $10 \mathrm{HD}$ patients, in 5 out of the 19 RTX patients, and in 1 out of 10 healthy controls. No differences were observed in baseline clinical parameters between the patients with and without a CHRS below 3 $\mathrm{ms} / \mathrm{mmHg}$. Noteworthy, CHRS was impaired in all 4 diabetic patients. Comparisons of CHRS according to medication directly related to the cardiovascular system (alpha-blockers, beta-blockers, Ca-antagonists,
ACE-inhibitors/AT-II-antagonists) or history (hypertension, coronary artery disease) failed to reveal any significant difference within and between the study groups.

In the RTX patients, pharmacological treatment with cyclosporine resulted in a decreased CHRS $(<3$ $\mathrm{ms} / \mathrm{mmHg}$ ) in 8 out of the 10 patients. The mean CHRS in this subgroup $(\mathrm{n}=10)$ was $2.8 \pm 0.5 \mathrm{~ms} / \mathrm{mm}$ Hg. The CHRS in the RTX patients without cyclosporine medication was decreased in 4 out of the 9 patients; three of the 4 patients being medicated with Ca-antagonists. The mean CHRS in this subgroup (n =9) was $4.7 \pm 0.3 \mathrm{~ms} / \mathrm{mmHg}$. The difference between the RTX patients with and without cyclosporine, however, failed to reach statistical significance $(P=0.13)$ probably because of a small sample size and a large scatter of data. Kidney function did not differ between these RTX sub-groups.

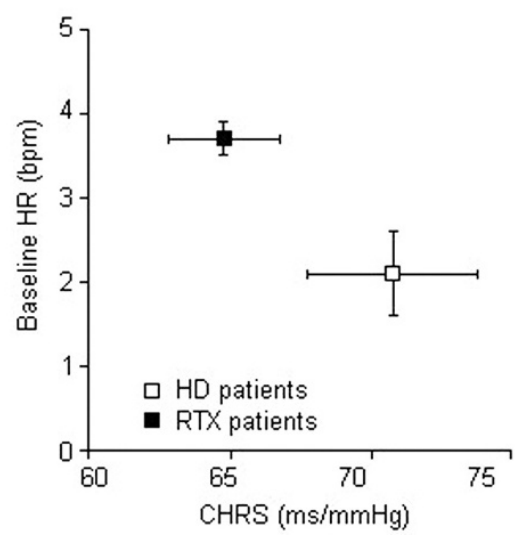

Fig. 2. Baseline heart rate (HR) was higher in HD patients as compared with RTX patients; being accompanied by impaired CHRS (both $\mathrm{P}<0.05)$. Values are means \pm SE.

\section{Relationship BetweEN SinUs Node AND Chemosensory ACtivity}

The difference in CHRS between the HD and RTX patients was accompanied by a lower baseline heart rate in the RTX patients (Fig. 2). By contrast, time-do-

Table 2. Heart Rate Variability in HD and RTX patients.

\begin{tabular}{lcc}
\hline & $\begin{array}{c}\text { HD Patients } \\
(\mathrm{n}=10)\end{array}$ & $\begin{array}{c}\text { RTX Patients } \\
(\mathrm{n}=10)\end{array}$ \\
\hline SDNN, ms & $99 \pm 18$ & $111 \pm 14^{*}$ \\
SDANN, ms & $92 \pm 17$ & $105 \pm 13^{*}$ \\
RMSSD, ms & $14 \pm 3$ & $21 \pm 3^{*}$ \\
Triangular index & $24 \pm 4$ & $29 \pm 4^{*}$ \\
\hline
\end{tabular}

RTX, patients after kidney transplantation; HD, patients on maintenance hemodialysis; SD, standard deviation; SDNN, 24-h SD of normal R-R intervals; SDANN, SD of the average normal R-R interval for all 5-min segments of a 24-h ECG recording; RMSSD, the square root of the mean of the squared differences between adjacent normal R-R intervals. Values are means \pm SE. $* \mathrm{P}<0.05$ 
main HRV parameters indicated a relative predominance of sympathetic activation in HD patients as compared with RTX patients (Table 2). On multiple regression analysis, RMSSD was the only independent predictor of an impaired hyperoxic CHRS ( $\mathrm{P}=0.03$, $\left.\mathrm{r}^{2}=0.59\right)$.

\section{Discussion}

The key findings of the present study are as follows: (1) Peripheral chemosensors' desensitization characteristics after kidney transplantation are blunted as compared with healthy controls and tend to be higher as compared with patients on maintenance hemodialysis, which seems to be related to co-morbidities and medication; and (2) Our findings indicate that chemosensory activity following kidney transplantation is related to cardiac autonomic control.

Several studies in animal models of renal failure suggest that excitation of renal afferent nerves results in increased efferent sympathetic nerve discharge [12]. This might in part explain the deteriorated chemosensory function in our patients. This is supported by the observation that the peripheral chemosensory activity following kidney transplantation was related to indirect measures of cardiac autonomic control, in particular, heart rate variability. Rubinger et al [6] found that successful transplantation corrected HRV abnormalities in most patients, suggesting that the autonomic dysfunction of uremia is caused by humoral factors reversed by the normalization of the renal function. However, direct measures of neural activity are needed. HRV reflects the fidelity with which post-junctional sinoatrial receptors respond to oscillations in sympathetic and vagal nerve discharge, rather than the absolute magnitude of neurotransmitter released.

High sympathetic drive in transplant recipients has been linked to cyclosporine and tacrolimus medications. It has also been suggested that the increase in blood pressure observed in kidney transplant patients is mediated by cyclosporine-induced sympatho-excitation. Of note, Hausberg et al [13] found that the withdrawal of cyclosporine in renal transplant patients, receiving relatively low doses of cyclosporine, resulted in a substantial decrease in blood pressure. However, peripheral muscle sympathetic nerve activity and norepinephrine did not change. Therefore, additional studies including both direct and indirect characterization of chemosensory function and sympathetic nerve activity (before/after kidney transplantation) are necessary to clarify this issue $[14,15]$.

There are several limitations of the present study that needs to be addressed: (1) We assessed chemosenory function in a relatively small number of patients with various causes of kidney disease; (2) We did not study RTX patients who had undergone bilateral nephrectomy. This is important since the cardiac sympathetic neural tone might be influenced by the diseased native kidneys despite correction of uremia; (3) We did not measure CHRS in the same patients before and after kidney transplantation; (4) HRV time-domain measures obtained by ambulatory ECG is confounded by daily activities, circadian changes, and medications used; (5) We did not measure HRV in healthy controls. Regarding the literature, HRV in our RTX patients seems still to be impaired which might in part explain the differences in CHRS between RTX patients and healthy controls; (6) We did not use the more sensitive, but at least more invasive MIBG, to characterize cardiac neural activity; and (7) Autonomic balance is maintained by multiple complex interacting reflex mechanisms, including arterial baroreflex, peripheral chemoreflex, central chemoreflex, and pulmonary stretch reflex. Further investigations using more sophisticated methods (e.g., cardiac and/or renal catecholamine spillover; direct measurement of sympathetic nerve activity) to characterize sympathetic activation are needed to explore the exact mechanisms of sympathetic chemosensory regulation following kidney transplantation.

\section{Conclusions and Future Perspectives}

Desensitization characteristics of peripheral chemosensors are deteriorated in ESRD and seem to be partly restored after kidney transplantation. In line with previous reports showing that tonic activation of excitatory chemoreflex afferents contributes to increased systemic efferent sympathetic activity in HD patients [16] and that kidney transplantation improves cardiorespiratory reflex measures of autonomic function [17-19] our findings suggest that chemosensory activity following kidney transplantation is related to cardiac autonomic control. Noteworthy, functional chemoreflex testing might only be useful to characterize the time course and extent of sympathetic activation in selected patients due to existing co-morbidities and medication in this population. Whether and to what extent neurohumoral modulation can reduce elevated sympathetic nerve activity and thereby restore chemosensory function in selected RTX patients needs to be explored. In the future, this might be useful to monitor therapeutic concepts targeting neurohumoral modulation in these patients.

Acknowledgments: C.M. is the owner of a research grant from the Hans-und-Gerti-Fischer-Stiftung.

Conflicts of interest: No conflicts of interests were declared by the authors in relation to this article.

\section{REFERENCES}

1. Wirta O, Pasternack A, Mustonen J, Laippala P. Renal and cardiovascular predictors of 9-year total and sudden cardiac mortality in non-insulin-dependent diabetic subjects. Nephrol Dial Transplant 1997; 12: 2612-7.

2. Converse Jr. RL, Jacobsen TN, Toto RD, Jost CMT, Cosentino F, Fouad-Tarazi F, Victor RG. Sympathetic overactivity in patients with chronic renal failure. N Engl J Med 1992; 327: 1912-8.

3. Ligtenberg G, Blankestijn PJ, Oey PL, Klein IH, Dijkhorst-Oei LT, Boomsma F, Wieneke GH, van Huffelen AC, Koomans HA. Reduction of sympathetic hyperactivity by enalapril in patients with chronic renal failure. N Engl J Med 1999; 340: 1321-8.

4. Neumann J, Ligtenberg G, Klein II, Koomans HA, Blankestijn PJ. Sympathetic hyperactivity in chronic kidney disease: pathogenesis, clinical relevance, and treatment. Kidney Int 2004; 65: 1568-76. 
5. Cashion AK, Hathaway DK, Milstead EJ, Reed L, Gaber AO. Changes in patterns of 24-hr heart rate variability after kidney and kidney-pancreas transplant. Transplantation 1999; 68: 1846-50.

6. Rubinger D, Sapoznikov D, Pollak A, Popovtzer MM, Luria MH. Heart rate variability during chronic hemodialysis and after renal transplantation: studies in patients without and with systemic amyloidosis. J Am Soc Nephrol 1999; 10: 1972-81.

7. Meyer C, Rana OR, Saygili E, Ozüyaman B, Latz K, Rassaf T, Kelm M, Schauerte P. Hyperoxic chemoreflex sensitivity is impaired in patients with neurocardiogenic syncope. Int J Cardiol 2010; 142: 38-43.

8. Levey AS, Bosch JP, Lewis JB, Greene T, Rogers N, Roth D. A more accurate method to estimate glomerular filtration rate from serum creatinine: A new prediction equation. Modification of Diet in Renal Disease Study Group. Ann Intern Med 1999; 130: 461-70.

9. Meyer C, Heiss C, Drexhage C, Kehmeier ES, Balzer J, Mühlfeld A, Merx MW, Lauer T, Kühl H, Floege J, Kelm M, Rassaf T. Hemodialysis-induced release of hemoglobin limits nitric oxide bioavailability and impairs vascular function. J Am Coll Cardiol 2010; 55: 454-9.

10. Hennersdorf MG, Hillebrand S, Perings C, Strauer BE. Chemoreflex sensitivity in chronic heart failure patients. Eur J Heart Fail 2001; 3: 679-84.

11. Hennersdorf MG, Niebch V, Perings C, Strauer BE. Chemoreflex sensitivity as a predictor of arrhythmia relapse in ICD recipients. Int J Cardiol 2002; 86: 169-75.

12. Hausberg M, Kosch M, Harmelink P, Barenbrock M, Hohage $\mathrm{H}$, Kisters $\mathrm{K}$, Dietl KH, Rahn KH. Sympathetic nerve activity in end-stage renal disease. Circulation 2002; 106: 1974-9.

13. Hausberg M, Lang D, Levers A, Suwelack B, Kisters K, Tokmak F, Barenbrock M, Kosch M. Sympathetic nerve activity in renal transplant patients before and after withdrawal of cyclosporine. J Hypertens 2006; 24: 957-64.
14. Meyer C, Rana OR, Saygili E, Gemein C, Becker M, Nolte KW, Weis J, Schimpf T, Knackstedt C, Mischke K, Hoffmann R, Kelm M, Pauza D, Schauerte P. Augmentation of left ventricular contractility by cardiac sympathetic neural stimulation. Circulation 2010; 121: 1286-94.

15. Somers VK, Mark AL, Abboud FM. Interaction of baroreceptor and chemoreceptor reflex control of sympathetic nerve activity in normal humans. J Clin Invest 1991; 87: 1953-7.

16. Hering D, Zdrojewski Z, Krol E, Kara T, Kucharska W, Somers VK, Rutkowski B, Narkiewicz K. Tonic chemoreflex activation contributes to the elevated muscle sympathetic nerve activity in patients with chronic renal failure. J Hypertens 2007; 25: 157-61.

17. Steinberg AA, Mars RL, Goldman DS, Percy RF. Effect of end-stage renal disease on decreased heart rate variability. Am J Cardiol 1998; 82: 1156-8, A10.

18. Tong YQ, Hou HM. Alteration of heart rate variability parameters in nondiabetic hemodialysis patients. Am J Nephrol 2007; 27: 63-9.

19. Tory K, Suveges Z, Horvath E, Bokor E, Sallay P, Berta K, Szabo A, Tulassay T, Reusz GS. Autonomic dysfunction in uremia assessed by heart rate variability. Pediatr Nephrol 2003; 18: 1167-71.

Address for correspondence:

Christian Meyer, M.D., M.A.

Heinrich-Heine-University Duesseldorf

Department of Medicine

Division of Cardiology, Pulmonology and Vascular Medicine Moorenstr. 5

40225 Duesseldorf

Phone: +492118118800

Fax: +49 2118118812

E-mail: Christian.Meyer@med.uni-duesseldorf.de 